\title{
Integrable models for confined fermions: applications to metallic grains
}

\author{
Luigi Amico $^{\mathrm{a}, \mathrm{b}}$, Antonio Di Lorenzo ${ }^{\mathrm{a}, \mathrm{b}}$, and \\ Andreas Osterloh ${ }^{\mathrm{a}, \mathrm{b}}$ \\ ${ }^{a}$ Dipartimento di Metodologie Fisiche e Chimiche (DMFCI), Università di \\ Catania, viale A. Doria 6, I-95125 Catania, Italy \\ ${ }^{\mathrm{b}}$ Istituto Nazionale per la Fisica della Materia, Unità di Catania, NEST-INFM
}

PACS N. 74.20.Fk, 02.30.Ik

\begin{abstract}
We study integrable models for electrons in metals when the single particle spectrum is discrete. The electron-electron interactions are BCS-like pairing, Coulomb repulsion, and spin exchange coupling. These couplings are, in general, nonuniform in the sense that they depend on the levels occupied by the interacting electrons. By using the realization of spin $1 / 2$-operators in terms of electrons the models describe spin $1 / 2$ models with nonuniform long range interactions and external magnetic field. The integrability and the exact solution arise since the model Hamiltonians can be constructed in terms of Gaudin models. Uniform pairing and the resulting orthodox model correspond to an isotropic limit of the Gaudin Hamiltonians. We discuss possible applications of this model to a single grain and to a system of few interacting grains.
\end{abstract}

\section{Introduction}

Recent advances in nanophysics allowed experimental investigations on isolated quantum dots and metallic nanoparticles[1]. Because of their small size, such systems have a discrete spectrum, which can be resolved through I$\mathrm{V}$ characteristic measurements. Besides potential technological applications, spectral analysis provides insight into the nature of the interactions in metals, comparable to the spectroscopy-based knowledge about the interactions in atoms and molecules[2]. This motivates the broad recent interest in various physical contexts: the spin orbit interaction was studied in mesoscopic $A l-A u$ systems[3]; various ferromagnetic properties were analyzed in experiments on $C o$ grains[4]; the experiments on $A l$ samples opened up debates on 
the crossover behavior of superconducting fluctuations, when the size of the sample is progressively reduced. The peculiarity of small grains and quantum dots is their small capacitance, which fixes the number of electrons in the system. In contrast, the characterization of many physical phenomena like e.g. phase transitions relies on quantities defined for infinite systems in the grand-canonical description. Therefore, the fingerprints of these phenomena, i.e. their characterizing physical quantities, must be identified at first[5]. Then, techniques suitable for systems in the canonical ensemble must be applied. Finally, for such small systems, approximations are difficult to control because of the presence of strong finite-size fluctuations and then, exact results are extremely valuable.

Many efforts have been devoted to the construction of theoretical models describing the physics of small metallic grains and quantum dots [6-9]. It has been argued that, due to the underlying disorder, the leading part of the electron-electron interaction reduces the Hamiltonian to a simple "universal" form (i.e. independent of the geometry of the system and the realization of the disorder), containing only the electrostatic interaction $E_{c}$, a BCS-like coupling $g$, and a spin-spin coupling $J$

$$
H_{U}=\sum_{i \sigma} \varepsilon_{i \sigma} n_{i \sigma}+E_{c} N^{2}-J \vec{S}^{2}-g K^{+} K^{-}
$$

where the $\varepsilon_{i}$ form the effective single-particle spectrum, $n_{i \sigma}$ is the electron number operator, $\vec{S}$ and $N$ are the total spin and number of electrons respectively. $K^{-}=\sum_{i} c_{i \downarrow} c_{i \uparrow}, K^{+}=K^{-\dagger}$ are the pair annihilation and creation operators. We point out that the couplings in (1) are independent of the involved single-particle levels; in this sense, they are uniform. Non-universal corrections to Hamiltonian (1) are of relative order $\delta / E_{T h}, E_{T h}$ being the Thouless energy and $\delta$ the average level spacing. $[6,7]$

Hamiltonian (1) has been used to attack a variety of physical phenomena[10]: equilibrium transport near degeneracy have been thoroughly investigated [6]; recently, Kurland et al. have shown that the paramagnetic phase is favored by the presence of disorder in the single-particle level spacing of the $\varepsilon_{i}$ [7].

For $g=0$, the Hamiltonian (1) describes a normal metallic grain with spinspin interaction. Its eigenstates are certain linear combinations of Fock states (eigenstates of $n_{i \sigma}$ ), such to be eigenstates of $\vec{S}^{2}$ as well; they are obtained applying the angular momentum lowering operator to the highest weight state. For $g \neq 0$ the exact solution of $H_{U}$ was found by Richardson and Sherman (RS) [11] using techniques close in spirit to the coordinate Bethe Ansatz (BA). By expressing $H_{U}$ in terms of commuting integrals of motion in Refs. $[12,13]$ (see also Ref. [14]), it was demonstrated that this Hamiltonian is also integrable. In fact, the exact BA eigenstates of $H_{U}$ have been successfully employed in the recent literature $[5,15]$. 
Non-equilibrium transport experiments [16] show that the corrections $\mathcal{O}\left(\delta / E_{T h}\right)$ to Hamiltonian (1) become very important for ultra-small samples. The presence of resonance clusters in the tunneling conductivity through $\mathrm{Al}$ grains are experimental evidence of fluctuations in the electrostatic interaction; in quantum dots the universal Hamiltonian (or Constant Interaction model) predicts a "bimodal" distribution of the tunneling-peak spacing, while a distribution corresponding to a Gaussian Orthogonal Ensemble (GOE) is observed [6]. Further, it has been argued that fluctuations in the spin pairing should influence the threshold for the mesoscopic Stoner instability, which could be observed in future experiments[8]. The presence of fluctuations leads to nonuniform couplings in the Hamiltonian, i.e. couplings whose strength depends on the two interacting levels. Not all nonuniform couplings yield an exactly solvable Hamiltonian; of course, demanding solvability results in restrictions on the admitted form of the interactions.

The aim of the present work is to explore the possible forms of the couplings compatible with the exact solvability of the corresponding models. We could not find[17] solvable generalizations of $H_{U}$ starting from a quadratic bosonic model, as done by RS. The strategy we adopt, instead, consists in generalizing the procedure of Ref. [12], namely constructing the Hamiltonian of the system in terms of Gaudin Hamiltonians. By means of the integrability and the exact solvability of the latter the exact solution of the model is obtained. For uniform couplings the integrals of motion are tightly related to the isotropic Gaudin Hamiltonians; for nonuniform couplings to the anisotropic Gaudin Hamiltonians. The models arising from this procedure include certain nonuniform interactions, which could account for corrections to the universal Hamiltonian due to e.g. disorder fluctuations. Namely:

$$
H=\sum_{i \sigma} \varepsilon_{i \sigma} n_{i \sigma}+\sum_{i j} U_{i j} n_{i} n_{j}-\sum_{i j} g_{i j} c_{i \uparrow}^{\dagger} c_{i \downarrow}^{\dagger} c_{j \downarrow} c_{j \uparrow}-\sum_{i j} J_{i j} \vec{S}_{i} \cdot \vec{S}_{j},
$$

where $U_{i j}, g_{i j}$ and $J_{i j}$ are the nonuniform generalizations of $E_{c}, g$ and $J$ in Eq.(1), respectively. This class of models can also be interpreted as describing systems of several coupled grains. Then, the coupling constants necessarily have to differ significantly when describing intra- and inter-grain interaction respectively. Other possible applications may be found in nuclear physics, where the nonuniformity of the couplings is notable.

This article is structured as follows: In section 2, we review how to derive the form of the Hamiltonian describing small grains, including the effects of disorder. Then we will rewrite the Hamiltonian in terms of spin and charge realizations of $\mathrm{su}(2)$. This will shed light on its structure, and lead to useful simplifications in the diagonalization procedure. In section 3, we sketch the solution of the universal part of the Hamiltonian and present the isotropic Gaudin Hamiltonians and their connection with the integrals of motion. In section 4, we construct integrable Hamiltonians by means of anisotropic Gaudin 
Hamiltonians. This class of Hamiltonians goes beyond the universal model with constant couplings. In general it is quadrilinear in the pseudo-spin operators. The tri- and quadrilinear terms can be eliminated by a suitable choice of parameters. In section 5, we discuss regimes of the interactions for exemplary choices of the parameters. Section 6 is dedicated to concluding remarks. In the appendix, we review some exact results obtained by Gaudin.

\section{The model Hamiltonian}

In 2nd quantization, the Hamiltonian for $N$ particles in a confining potential $\hat{V}_{1}=\sum_{a} V\left(\hat{\mathbf{r}}_{a}\right)$ and a (spin-independent) electron-electron interaction $\hat{V}_{2}=$ $\frac{1}{2} \sum_{a \neq b} v\left(\hat{\mathbf{r}}_{a}-\hat{\mathbf{r}}_{b}\right), a, b \in\{1, \ldots, N\}$ is

$$
\begin{aligned}
H & =\sum_{a} \frac{\hat{\mathbf{p}}_{a}^{2}}{2 m}+\hat{V}_{1}+\hat{V}_{2}=\sum_{i \sigma} \tilde{\varepsilon}_{i \sigma} n_{i \sigma}+\sum_{i j k l} \sum_{\sigma \tau} M_{k l}^{i j} c_{i \sigma}^{\dagger} c_{j \tau}^{\dagger} c_{l \tau} c_{k \sigma}, \\
M_{k l}^{i j} & =\frac{1}{2} \int d \mathbf{r} d \mathbf{r}^{\prime} \phi_{i}^{*}(\mathbf{r}) \phi_{j}^{*}\left(\mathbf{r}^{\prime}\right) v\left(\mathbf{r}-\mathbf{r}^{\prime}\right) \phi_{l}\left(\mathbf{r}^{\prime}\right) \phi_{k}(\mathbf{r}),
\end{aligned}
$$

where $\tilde{\varepsilon}_{i \sigma}$ and $\phi_{i}(\mathbf{r}) \chi_{i}(\sigma), i \in\{1, \ldots, \Omega\}:=I$ are the eigenvalues and eigenfunctions of the single particle Hamiltonian $\left(\chi(\sigma)\right.$ is the spinor basis); $c_{i \sigma}^{\dagger}, c_{i \sigma}$ are electronic creation and annihilation operators. The disorder average reduces the leading part $\bar{M}$ of the matrix elements $M_{k l}^{i j}$ to be "uniform-diagonal"; i.e. only elements corresponding to pairwise equal indices are non-zero and constant: $\bar{M}_{i j}^{i j}:=\alpha, \bar{M}_{j i}^{i j}:=\beta$ and $\bar{M}_{j j}^{i i}:=\gamma$ for $i \neq j, \bar{M}_{i i}^{i i}:=\omega$. We can rewrite the interaction matrix as

$$
\begin{aligned}
M_{k l}^{i j}= & \delta_{i k} \delta_{j l}\left(1-\delta_{i j}\right) \alpha+\delta_{i l} \delta_{j k}\left(1-\delta_{i j}\right) \beta+\delta_{i j} \delta_{k l}\left(1-\delta_{i k}\right) \gamma \\
& +\delta_{i j} \delta_{j k} \delta_{k l} \omega+\delta M_{k l}^{i j}
\end{aligned}
$$

the (non-universal) fluctuations $\delta M_{k l}^{i j}$ are such that $\overline{\delta M_{k l}^{i j} \delta M_{k l}^{i j}}=\mathcal{O}\left(\delta^{2} / E_{T h}^{2}\right)$, i.e. the corrections are of relative order $\delta / E_{T h}$. Inserting expression (4) in the Hamiltonian (3), we obtain $H=H_{U}+\delta H$, where

$$
\begin{aligned}
H_{U}= & \sum_{i \sigma}\left(\tilde{\varepsilon}_{i \sigma}+\delta M_{i i}^{i i}\right) n_{i \sigma}+\sum_{i \neq j} \sum_{\sigma \tau}\left[\alpha n_{i \sigma} n_{j \tau}+\beta c_{i \sigma}^{\dagger} c_{j \tau}^{\dagger} c_{i \tau} c_{j \sigma}+\gamma c_{i \sigma}^{\dagger} c_{i \tau}^{\dagger} c_{j \tau} c_{j \sigma}\right] \\
& +\sum_{i} \sum_{\sigma \tau} \omega c_{i \sigma}^{\dagger} c_{i \tau}^{\dagger} c_{i \tau} c_{i \sigma} \\
= & \sum_{i \sigma} \varepsilon_{i \sigma} n_{i \sigma}+\sum_{i j}\left[\alpha n_{i} n_{j}-\beta \sum_{\sigma}\left(c_{i \sigma}^{\dagger} c_{i \bar{\sigma}} c_{j \bar{\sigma}}^{\dagger} c_{j \sigma}+n_{i \sigma} n_{j \sigma}\right)+2 \gamma c_{i \uparrow}^{\dagger} c_{i \downarrow}^{\dagger} c_{j \downarrow} c_{j \uparrow}\right]
\end{aligned}
$$




$$
\begin{aligned}
& +2(\omega-\alpha-\beta-\gamma) N_{\text {pairs }} \\
\delta H= & \sum_{i j}\left[\delta M_{i j}^{i j} n_{i} n_{j}-\delta M_{j i}^{i j} \sum_{\sigma}\left(c_{i \sigma}^{\dagger} c_{i \bar{\sigma}} c_{j \bar{\sigma}}^{\dagger} c_{j \sigma}+n_{i \sigma} n_{j \sigma}\right)+2 \delta M_{j j}^{i i} c_{i \uparrow}^{\dagger} c_{i \downarrow}^{\dagger} c_{j \downarrow} c_{j \uparrow}\right] \\
& -\sum_{i} 4 \delta M_{i i}^{i i} n_{i \uparrow} n_{i \downarrow} \\
& +\sum_{i j k l} \sum_{\sigma \tau}\left(1-\delta_{i j} \delta_{k l}\right)\left(1-\delta_{i k} \delta_{j l}\right)\left(1-\delta_{i l} \delta_{j k}\right) M_{k l}^{i j} c_{i \sigma}^{\dagger} c_{j \tau}^{\dagger} c_{l \tau} c_{k \sigma},
\end{aligned}
$$

where $n_{i}=\sum_{\sigma} n_{i \sigma}$ is the number of electrons in the $i$-th level-pair, $\bar{\sigma}=-\sigma$, $N_{\text {pairs }}=\sum_{i} n_{i \uparrow} n_{i \downarrow}$ is the number of pairs, and $\varepsilon_{i \sigma}=\tilde{\varepsilon}_{i \sigma}-\alpha+2 \beta+\delta M_{i i}^{i i}$. Introducing the spin operators

$$
S_{j}^{+}=c_{j \uparrow}^{\dagger} c_{j \downarrow} \quad, \quad S_{j}^{-}=\left(S_{j}^{+}\right)^{\dagger} \quad, \quad S_{j}^{z}=\frac{1}{2}\left(n_{j \uparrow}-n_{j \downarrow}\right),
$$

we have $n_{i \uparrow}=\frac{1}{2} n_{i}+S_{i}^{z}, n_{i \downarrow}=\frac{1}{2} n_{i}-S_{i}^{z}$, and then:

$$
\begin{aligned}
H_{U}= & \sum_{i \sigma} \varepsilon_{i \sigma} n_{i \sigma}+\sum_{i j}\left[\left(\alpha-\frac{\beta}{2}\right) n_{i} n_{j}-\beta \vec{S}_{i} \cdot \vec{S}_{j}+2 \gamma c_{i \uparrow}^{\dagger} c_{i \downarrow}^{\dagger} c_{j \downarrow} c_{j \uparrow}\right] \\
& +2(\omega-\alpha-\beta-\gamma) N_{\text {pairs }} \\
\delta H= & \sum_{i j}\left[\left(\delta M_{i j}^{i j}-\frac{1}{2} \delta M_{j i}^{i j}\right) n_{i} n_{j}-\delta M_{j i}^{i j} \vec{S}_{i} \cdot \vec{S}_{j}+2 \delta M_{j j}^{i i} c_{i \uparrow}^{\dagger} c_{i \downarrow}^{\dagger} c_{j \downarrow} c_{j \uparrow}\right] \\
& -\sum_{i} 4 \delta M_{i i}^{i i} n_{i \uparrow} n_{i \downarrow} \\
& +\sum_{i j k l} \sum_{\sigma \tau}\left(1-\delta_{i j} \delta_{k l}\right)\left(1-\delta_{i k} \delta_{j l}\right)\left(1-\delta_{i l} \delta_{j k}\right) M_{k l}^{i j} c_{i \sigma}^{\dagger} c_{j \tau}^{\dagger} c_{l \tau} c_{k \sigma} .
\end{aligned}
$$

From $H_{U}$ we recover, up to a constant of motion, the universal Hamiltonian (1), defining $E_{c} \equiv \alpha-\beta / 2, J \equiv \beta$ and $g \equiv-2 \gamma$. This constant of motion is relevant to determine the ground state properties.

The Hamiltonian studied within the present paper is the sum of the uniform part (6) and the first line of the nonuniform correction (7):

$$
\begin{aligned}
H= & \sum_{i} 2 \zeta_{i} S_{i}^{z}-\sum_{i j} J_{i j} \vec{S}_{i} \cdot \vec{S}_{j} \\
& +\sum_{i} \xi_{i} n_{i}+\sum_{i j} U_{i j} n_{i} n_{j}-\sum_{i j} g_{i j} c_{i \uparrow}^{\dagger} c_{i \downarrow}^{\dagger} c_{j \downarrow} c_{j \uparrow},
\end{aligned}
$$

where we defined $2 \zeta_{i}=\varepsilon_{i \uparrow}-\varepsilon_{i \downarrow}, 2 \xi_{i}=\varepsilon_{i \uparrow}+\varepsilon_{i \downarrow}$. It can be written in a more perspicuous way introducing the operators

$$
K_{j}^{+}=c_{j \uparrow}^{\dagger} c_{j \downarrow}^{\dagger}, \quad K_{j}^{-}=\left(K_{j}^{+}\right)^{\dagger}, \quad K_{j}^{z}=\frac{1}{2}\left(n_{j \uparrow}+n_{j \downarrow}-1\right),
$$


which are generators of the charge $s u(2):\left[K_{i}^{ \pm}, K_{j}^{z}\right]= \pm \delta_{i j} K_{i}^{ \pm},\left[K_{i}^{+}, K_{j}^{-}\right]=$ $2 \delta_{i j} K_{i}^{z}$. The charge $s u(2)$ is orthogonal to the spin $s u(2)$, i.e. their generators have the property $\left[S_{j}^{a}, K_{k}^{b}\right]=0$. In terms of the spin and charge $s u(2)$ operators, the Hamiltonian (8) can be finally written as

$$
\begin{aligned}
H & =H_{S}+H_{K}+E_{0} \\
H_{S} & =\sum_{i} 2 \zeta_{i} S_{i}^{z}-\sum_{i j} J_{i j} \vec{S}_{i} \cdot \vec{S}_{j} \\
H_{K} & =\sum_{i} 2 \eta_{i} K_{i}^{z}+4 \sum_{i j} U_{i j} K_{i}^{z} K_{j}^{z}-\frac{1}{2} \sum_{i j} g_{i j}\left(K_{i}^{+} K_{j}^{-}+K_{i}^{-} K_{j}^{+}\right),
\end{aligned}
$$

where $2 \eta_{i}=2 \xi_{i}+4 \sum_{j} U_{i j}-g_{i i}$ and $E_{0}=\sum_{i} \xi_{i}+\sum_{i j} U_{i j}$.

In what follows, we refer to $H_{S}$ as spin Hamiltonian, and to $H_{K}$ as charge Hamiltonian. They belong to the class of integrable models we find in section 4 .

We note that $\left[H, S^{z}\right]=\left[H, K^{z}\right]=0$. Hence we can diagonalize the Hamiltonian in the subspace of fixed number of pairs $N_{K}$ and up spins $N_{S}$. Because of the orthogonality of the spin and charge $s u(2)$, it follows that $\left[H_{K}, H_{S}\right]=0$ and in particular $\left[H_{S}, K_{i}^{ \pm}\right]=\left[H_{K}, S_{i}^{ \pm}\right]=0$. Thus $H_{S}$ and $H_{K}$ are degenerate respect to the action of $K_{i}^{ \pm}$and $S_{i}^{ \pm}$respectively. Therefore, the Hilbert space $\mathcal{H}$ can be split into $\mathcal{H}_{S}$ and $\mathcal{H}_{K}$ created by the action of $N_{S} S_{i}^{+}$and $N_{K} K_{i}^{+}$on the vacua $|0\rangle_{S}$ and $|0\rangle_{K}$ respectively. The spin vacuum $|0\rangle_{S}=|\downarrow, \ldots, \downarrow\rangle$ is the state in which all electrons of the singly occupied level-pairs have spin down; the charge vacuum $|0\rangle_{K}$ is the state in $\mathcal{H}_{K}$ with all level-pairs empty. Namely, $\mathcal{H}_{K}$ consists of both the doubly occupied and empty level-pairs which we label with the index set $I_{K}\left(\left|I_{K}\right|=\Omega_{K}\right)$, and $\mathcal{H}_{S}$ consists of the singly occupied levels-pairs, which we label by the index set $I_{S}=I \backslash I_{K}\left(\left|I_{S}\right|=\Omega_{S}\right)$. This factorization of the Hilbert space was called the "blocking of singly occupied levels" when considering the charge Hamiltonian only[11], and it reduces the problem of finding the spectrum of $H$ to the diagonalization of $H_{S}$ and $H_{K}$ in $\mathcal{H}_{S}$ and $\mathcal{H}_{K}$, respectively.

\section{Uniform couplings}

In this section, we recast the known results[5,11,12] for the universal Hamiltonian in a uniform magnetic field in the present frame. In this case, having decomposed the universal model (1) as in formula (10), the spin and charge Hamiltonians are

$$
H_{S}=2 \zeta S^{z}-J \vec{S}^{2}
$$




$$
H_{K}=\sum_{i} 2 \eta_{i} K_{i}^{z}-\frac{g}{2} \sum_{i j}\left(K_{i}^{+} K_{j}^{-}+K_{i}^{-} K_{j}^{+}\right)+4 E_{c}\left(\sum_{i} K_{i}^{z}\right)^{2},
$$

and $E_{0}=\sum_{i} \xi_{i}+\Omega_{K}^{2} E_{c}$, while the parameters are $\zeta=\mu_{B} B$ and $2 \eta_{i}=2 \xi_{i}+$ $4 E_{c} \Omega_{K}-g$. The eigenstates $|\Psi\rangle_{N_{S}}=\left(S^{+}\right)^{N_{S}}|0\rangle_{S}$ of $H_{S}$ are common eigenstates of the total spin $\vec{S}^{2}$ and $S^{z}$ with eigenvalues $E_{N_{S}}=2 \mu_{B} B\left(N_{S}-\Omega_{S} / 2\right)-J S(S+$ $1)$.

$H_{K}$ can be diagonalized through coordinate [11] or algebraic [18] BA. Due to its integrability, it suffices to diagonalize its integrals of motion $\tau_{i}$. Indeed, $H_{K}$ can be written as

$$
\begin{gathered}
H_{K}=\sum_{i} 2 \eta_{i} \tau_{i}+\left(4 E_{c}+g\right) \sum_{i j} \tau_{i} \tau_{j}+\text { const. } \\
\tau_{i}(\mathbf{K})=K_{i}^{z}-g \sum_{j \neq i} \frac{\vec{K}_{i} \cdot \vec{K}_{j}}{\eta_{i}-\eta_{j}}:=K_{i}^{z}+\Xi_{i} .
\end{gathered}
$$

where $\Xi_{i}$ are the isotropic Gaudin Hamiltonians (see also the appendix). With a straightforward generalization of Gaudin's results[19,20], one recovers eigenstates and eigenvalues of $\tau_{i}$, and from these the eigenvalues $E_{N_{K}}$ of $H_{K}$

$$
\begin{aligned}
\left|\Psi_{N_{K}}\right\rangle & =\prod_{\alpha} \sum_{i} \frac{K_{i}^{+}}{E_{\alpha}-2 \xi_{i}}|0\rangle_{K} \\
E_{N_{K}} & =\sum_{\alpha} E_{\alpha}+E_{c}\left(2 N_{K}-\Omega_{K}\right)^{2} .
\end{aligned}
$$

The quasi-energies $E_{\alpha}$ are solutions of the RS equations

$$
-\frac{1}{g}+\sum_{j} \frac{1}{2 \xi_{j}-E_{\alpha}}=2 \sum_{\beta}^{\prime} \frac{1}{E_{\beta}-E_{\alpha}} \quad \alpha=1 \ldots N_{K} .
$$

Here and in the rest of the paper, a primed sum means that coincidences of indices are avoided, e.g. $\sum_{\alpha}^{\prime} f(\alpha, \beta):=\sum_{\substack{\alpha \neq \beta \\ \alpha \neq \beta}} f(\alpha, \beta)$.

\section{4 nonuniform couplings}

In this section we consider the following class of models, which includes Hamiltonian (10),

$$
\tilde{H}_{K}=\sum_{i} 2 \eta_{i} K_{i}^{z}-\frac{1}{2} \sum_{i j} g_{i j}\left(K_{i}^{+} K_{j}^{-}+K_{i}^{-} K_{j}^{+}\right)+4 \sum_{i j} U_{i j} K_{i}^{z} K_{j}^{z}
$$




$$
\tilde{H}_{S}=\sum_{i} 2 \zeta_{i} S_{i}^{z}-\frac{1}{2} \sum_{i j} J_{i j}\left(S_{i}^{+} S_{j}^{-}+S_{i}^{-} S_{j}^{+}\right)-\sum_{i j} J_{i j}^{z} S_{i}^{z} S_{j}^{z},
$$

We prove the integrability of this class for a particular choice of the coupling constants (Eq. (20) below), and find its exact solution. The proof of integrability is constructive and performed for the spin and charge Hamiltonian separately (see the discussion at the end of section 2). We summarize the results of subsections $\mathrm{A}$ and $\mathrm{B}$.

We find that $H_{K}$ and $H_{S}$ are integrable if the couplings are

$$
\left.\begin{array}{c}
g_{i j}=-\frac{p u\left(\eta_{i}-\eta_{j}\right)}{\sinh \left[p\left(u_{i}-u_{j}\right)\right]}, \\
4 U_{i j}=A+p u\left(\eta_{i}-\eta_{j}\right) \operatorname{coth}\left[p\left(u_{i}-u_{j}\right)\right], \\
J_{i j}=-\frac{q v\left(\zeta_{i}-\zeta_{j}\right)}{\sinh \left[q\left(v_{i}-v_{j}\right)\right]}, \\
J_{i j}^{z}=-q v\left(\zeta_{i}-\zeta_{j}\right) \operatorname{coth}\left[q\left(v_{i}-v_{j}\right)\right],
\end{array}\right\}
$$

where the parameters $A, u, v, u_{i}, \eta_{i}$ and $v_{i}$ are arbitrary real numbers, while $p$ and $q$ are imaginary or real. A quantitative discussion of the couplings for an exemplary and physically reasonable choice of parameters, is done in section 5 . We remark that no analog to $A$ occurs for the spin term. The reason is that we can express $H_{S}$ even without the bilinear term in the integrals of motion, and that $\sum_{i, j} \tau_{i}(\mathbf{S}) \tau_{j}(\mathbf{S})=\left(S^{z}\right)^{2}$ is an integral of motion due to a symmetry (the $S^{z}$-symmetry of the Hamiltonian) which is not related with integrability. For $H_{K}$, instead, this constant is needed to keep the electron-electron Coulomb interaction repulsive.

For every partition of the set $I$ in distinct subsets $I_{S}, I_{K}$, we obtain the eigenstates

$$
|\Psi\rangle=\prod_{\alpha=1}^{N_{K}} \sum_{i \in I_{K}} \frac{p K_{i}^{+}}{\sinh \left[p\left(\omega_{\alpha}-u_{i}\right)\right]} \prod_{\beta=1}^{N_{S}} \sum_{j \in I_{S}} \frac{q S_{j}^{+}}{\sinh \left[q\left(\nu_{\beta}-v_{j}\right)\right]}|0\rangle_{K} \otimes|0\rangle_{S}
$$

whose energy is $E=E_{N_{K}}+E_{N_{S}}+E_{0}$, where $E_{N_{K}}$ and $E_{N_{S}}$ are given by

$$
\begin{gathered}
E_{N_{K}}=p u \sum_{j \alpha} \eta_{j} \operatorname{coth}\left[p\left(\omega_{\alpha}-u_{j}\right)\right]-A N_{K}\left(\Omega_{K}-N_{K}\right)-E_{K} \\
E_{N_{S}}=p v \sum_{j \alpha} \zeta_{j} \operatorname{coth}\left[p\left(\nu_{\alpha}-v_{j}\right)\right]-\frac{1}{4} \sum_{i j} J_{i j}^{z}-\frac{1}{2} \sum_{i} J_{i i} .
\end{gathered}
$$


, where $E_{K}=\sum_{i}\left(\xi_{i}+\sum_{j} U_{i j}+2 \sum_{j \in I_{S}} U_{i j}\right)$. From here on, latin indices, when referring to the charge (spin) Hamiltonian, range in $I_{K}\left(I_{S}\right)$, and greek indices from 1 to $N_{K}\left(N_{S}\right)$. The parameters $\omega_{\alpha}, \nu_{\beta}$ must fulfill the following set of equations

$$
\begin{aligned}
& \frac{2}{u}+\sum_{j} p \operatorname{coth} p\left[\left(u_{j}-\omega_{\alpha}\right)\right]=2 \sum_{\beta}^{\prime} p \operatorname{coth}\left[p\left(\omega_{\beta}-\omega_{\alpha}\right)\right], \\
& \frac{2}{v}+\sum_{j} q \operatorname{coth} q\left[\left(v_{j}-\nu_{\alpha}\right)\right]=2 \sum_{\beta}^{\prime} q \operatorname{coth}\left[q\left(\nu_{\beta}-\nu_{\alpha}\right)\right] .
\end{aligned}
$$

For XXX-type coupling, present in the spin Hamiltonian (11), that is $J^{z} \equiv J$, the coupling has the form

$$
J_{i j}=-v \frac{\zeta_{i}-\zeta_{j}}{v_{i}-v_{j}} \quad \text { for } i \neq j
$$

the eigenstates are then

$$
\left|\Phi_{N_{S}}^{x x x}\right\rangle=\prod_{\beta} \sum_{j} \frac{S_{j}^{+}}{\nu_{\beta}-v_{j}}|0\rangle_{S}
$$

with eigenenergy

$$
E_{N_{S}}=\sum_{j \alpha} \frac{v \zeta_{i}}{\nu_{\alpha}-v_{i}}-\sum_{j} \zeta_{j}-\frac{1}{4} \sum_{i j} J_{i j}-\frac{1}{2} \sum_{i} J_{i i} .
$$

The quantities $\nu_{\beta}$ must fulfill the RS equations

$$
\frac{2}{v}+\sum_{j} \frac{1}{v_{j}-\nu_{\alpha}}=2 \sum_{\beta}^{\prime} \frac{1}{\nu_{\beta}-\nu_{\alpha}}
$$

Finally, for uniform Zeeman splitting $\zeta_{j} \equiv \mu_{B} B$, the eigenstates are still given by (27), but the Gaudin equations

$$
\sum_{j} \frac{1}{v_{j}-\nu_{\alpha}}=2 \sum_{\beta}^{\prime} \frac{1}{\nu_{\beta}-\nu_{\alpha}}
$$

have to be fulfilled instead of (29). The eigenenergy $E_{N_{S}}$ is then

$$
E_{N_{S}}=\sum_{i \alpha} \frac{v \phi_{i}}{\nu_{\alpha}-v_{i}}+\mu_{B} B\left(2 N_{S}-\Omega_{S}\right)-\frac{1}{4} \sum_{i j} J_{i j}-\frac{1}{2} \sum_{i} J_{i i}
$$


instead of (28), with arbitrary parameters $\phi_{i}$. The couplings are given by Eq.(26), replacing $\zeta_{i}$ with $\phi_{i}$.

\subsection{The charge Hamiltonian}

To construct an integrable model for non uniform couplings we generalize formula (14) to:

$$
\tilde{H}_{K}=\sum_{i} 2 \eta_{i} \tilde{\tau}_{i}+\sum_{i j} A_{i j} \tilde{\tau}_{i} \tilde{\tau}_{j}-\sum_{i} \frac{3}{4} \phi_{i}
$$

with real symmetric $A_{i j}$, wherein the operators $\tilde{\tau}_{i}$ are obtained by substituting the isotropic Gaudin models $\Xi_{i}$ in formula (15) by the anisotropic Gaudin Hamiltonians $\tilde{\Xi}_{j}(\boldsymbol{\sigma})$ (see also appendix)

$$
\begin{gathered}
\tilde{\Xi}_{j}(\boldsymbol{\sigma})=\sum_{k}^{\prime} w_{j k}^{a} \sigma_{j}^{a} \sigma_{k}^{a} . \\
\tilde{\tau}_{i}=K_{i}^{z}+\tilde{\Xi}_{i}(\mathbf{K}) .
\end{gathered}
$$

A sufficient condition for these operators to commute with each other is that the coefficients $w_{i j}^{\alpha}$ of $\tilde{\Xi}_{j}(\boldsymbol{\sigma})$ can be parametrized as $w_{i j}^{z} \equiv v_{i j}=p u \operatorname{coth}\left[p\left(u_{i}-u_{j}\right)\right]$, $w_{i j}^{x}=w_{i j}^{y} \equiv w_{i j}=p u / \sinh \left[p\left(u_{i}-u_{j}\right)\right]$. While $\left\{u_{i}\right\}, u$ are real, the parameter $p$ can be real or imaginary and switches between hyperbolic and trigonometric functions respectively[21]. The resulting Hamiltonian is

$$
\begin{aligned}
\tilde{H}_{K}= & \sum_{i} 2 \eta_{i} K_{i}^{z}-\frac{1}{2} \sum_{i j} g_{i j}\left(K_{i}^{+} K_{j}^{-}+K_{i}^{-} K_{j}^{+}\right)+4 \sum_{i j} U_{i j} K_{i}^{z} K_{j}^{z} \\
& +H_{3}+H_{4}
\end{aligned}
$$

with the couplings

$$
\begin{aligned}
g_{i j} & =-\frac{p u\left(\eta_{i}-\eta_{j}\right)}{\sinh \left[p\left(u_{i}-u_{j}\right)\right]}, \\
4 U_{i j} & =A_{i j}+p u\left(\eta_{i}-\eta_{j}\right) \operatorname{coth}\left[p\left(u_{i}-u_{j}\right)\right],
\end{aligned}
$$

The Hamiltonians $H_{3}$ and $H_{4}$ contain interactions up to tri- and quadri-linear in the charge $s u(2)$ operators: 


$$
\begin{aligned}
H_{3} & =\sum_{i j} A_{i j}\left(K_{i}^{z} \tilde{\Xi}_{j}(\mathbf{K})+\tilde{\Xi}_{i}(\mathbf{K}) K_{j}^{z}\right) \\
& =\sum_{i j k}^{\prime}\left[A_{i j}-A_{i k}\right]\left(v_{j k} K_{i}^{z} K_{j}^{+} K_{k}^{-}+w_{j k} K_{i}^{z} K_{j}^{z} K_{k}^{z}\right) \\
& +\frac{1}{2} \sum_{i j}^{\prime}\left[A_{i j}-A_{j j}\right] w_{i j} K_{i}^{z}, \\
H_{4} & =\sum_{i j} A_{i j} \tilde{\Xi}_{i}(\mathbf{K}) \tilde{\Xi}_{j}(\mathbf{K}) \\
& =\sum_{i j k l}^{\prime} \frac{1}{2}\left[A_{i k}-A_{i l}+A_{j l}-A_{j k}\right] v_{i j} w_{k l} K_{i}^{z} K_{j}^{z} K_{k}^{+} K_{l}^{-} \\
& +\frac{1}{4} \sum_{i j}^{\prime}\left\{2 A_{i j}-A_{i i}-A_{j j}\right\} w_{i j} v_{i j} K_{i}^{+} K_{i}^{-} \\
& +\frac{1}{4} \sum_{i j}^{\prime} \sum_{k l}^{\prime}\left\{A_{i k}-A_{j k}-A_{i l}+A_{k l}\right\} w_{i j} w_{k l} K_{i}^{+} K_{j}^{-} K_{k}^{+} K_{l}^{-} \\
& +\frac{1}{4} \sum_{i j}^{\prime} \sum_{k l}^{\prime}\left\{A_{i k}-A_{j k}-A_{i l}+A_{k l}\right\} v_{i j} v_{k l} K_{i}^{z} K_{j}^{z} K_{k}^{z} K_{l}^{z} .
\end{aligned}
$$

It is interesting to note that $H_{3}$ and $H_{4}$ vanish for $A_{i j}=A$, which can be also seen directly from equations (38) and (39), because of $\sum_{i} \Xi_{i}=0$ (see appendix). Another possible simplification can be achieved for $A_{i j}=\alpha_{i}+\alpha_{j}$, for which the overall effect of the second term in Eq.(32) is to renormalize the parameters $\eta_{j}$ :

$$
\tilde{H}_{K}=\sum_{j} 2\left(\eta_{j}+K^{z} \alpha_{j}\right) \tilde{\tau}_{j}
$$

We point out that the Coulomb term can be canceled choosing $A_{i j}=g_{i j} \cosh \left[p\left(u_{i}-u_{j}\right)\right][22]$. In $\mathcal{H}_{K}$, the eigenstates of $\tilde{\tau}_{i}(\mathbf{K})$, and hence of $\tilde{H}_{K}$, are

$$
\left|\Psi_{N_{K}}\right\rangle=\prod_{\alpha} \sum_{j} \frac{p K_{j}^{+}}{\sinh \left[p\left(\omega_{\alpha}-u_{j}\right)\right]}|0\rangle_{K} .
$$

The eigenvalues $\tilde{t}_{i}$ of $\tilde{\tau}_{i}(\mathbf{K})$ are given by

$$
\tilde{t}_{i}=\frac{u}{2} \sum_{\alpha} p \operatorname{coth}\left[p\left(\omega_{\alpha}-u_{i}\right)\right]-\frac{u}{4} \sum_{j}^{\prime} p \operatorname{coth}\left[p\left(u_{j}-u_{i}\right)\right]-\frac{1}{2}
$$

The quantities $\omega_{\alpha}$ must fulfill the equations

$$
\frac{2}{u}+\sum_{j} p \operatorname{coth} p\left[\left(u_{j}-\omega_{\alpha}\right)\right]=2 \sum_{\beta}^{\prime} p \operatorname{coth}\left[p\left(\omega_{\beta}-\omega_{\alpha}\right)\right] .
$$


We discuss the case of constant $A_{i j} \equiv A$. Then, the Hamiltonian simplifies and formula (32) becomes

$$
\begin{aligned}
\tilde{H}_{K} & =\sum_{i} 2 \eta_{i} \tilde{\tau}_{i}+A \sum_{i j} \tilde{\tau}_{i} \tilde{\tau}_{j}=\sum_{i} 2 \eta_{i} \tilde{\tau}_{i}+A\left(\sum_{i} \tilde{\tau}_{i}\right)^{2} \\
& =\sum_{i} 2 \eta_{i} \tilde{\tau}_{i}+A\left(K^{z}\right)^{2} .
\end{aligned}
$$

In this case $\tilde{H}_{K}$ is the Hamiltonian (12), provided the coupling constants in the latter are parametrized as in Eq. (37) for $A_{i j} \equiv A$. The eigenstates are still given by (41) with the eigenvalues

$$
E_{N_{K}}=p u \sum_{j \alpha} \eta_{j} \operatorname{coth}\left[p\left(\omega_{\alpha}-u_{j}\right)\right]-A N_{K}\left(\Omega_{K}-N_{K}\right)-E_{K}
$$

In the $p \rightarrow 0$ limit, which corresponds to the isotropic limit for the Gaudin Hamiltonians, the coupling constants become

$$
\begin{array}{cl}
g_{i j} & =-u\left(\eta_{i}-\eta_{j}\right) /\left(u_{i}-u_{j}\right), \\
& \text { for } i \neq j \\
4 U_{i j} & =A+u\left(\eta_{i}-\eta_{j}\right) /\left(u_{i}-u_{j}\right),
\end{array}
$$

The eigenstates and eigenvalues are

$$
\begin{aligned}
\left|\Psi_{N_{K}}\right\rangle & =\prod_{\alpha} \sum_{i} \frac{K_{i}^{+}}{\omega_{\alpha}-u_{i}}|0\rangle_{K} \\
E_{N_{K}} & =\sum_{i \alpha} \frac{\eta_{i}}{\omega_{\alpha}-u_{i}}-A N_{K}\left(\Omega_{K}-N_{K}\right)-E_{0},
\end{aligned}
$$

where $\omega_{\alpha}$ have to be solutions of the equations

$$
\frac{2}{u}+\sum_{j} \frac{1}{u_{j}-\omega_{\alpha}}=2 \sum_{\beta}^{\prime} \frac{1}{\omega_{\beta}-\omega_{\alpha}} .
$$

Uniform couplings $g_{i j}=g, U_{i j}=E_{c}$ are then obtained from a linear $u-\eta$ relation. Choosing $u_{j}=-2 \eta_{j} / g, \omega_{\alpha}=-E_{\alpha} / g$, and $u=2$, Eqs.(48) are the RS equations (17) (we recall that $e t a_{i}=\xi_{i}+$ const.). Setting $A=4 E_{c}+g$ as well, the Hamiltonian (12) is the BCS Hamiltonian (up to a constant). 


\subsection{The spin Hamiltonian}

We remark, that the procedure leading here to the exact solution of the spin Hamiltonian coincides with that applied to $H_{K}$, because the underlying algebraic structure is still $s u(2)$.

We study the Hamiltonian

$$
H_{S}^{x x z}=\sum_{i} 2 \zeta_{i} \tau_{i}-\frac{3}{4} J \Omega_{S}
$$

with $\tau_{i}=S_{i}^{z}+\tilde{\Xi}_{i}(\mathbf{S})$. Also here, the conservation of the $z$-component of the total spin restricts the anisotropy of the Gaudin models to the XXZ type. The Hamiltonian we get from Eq. (49) is

$$
H_{S}^{x x z}=\sum_{i} 2 \zeta_{i} S_{i}^{z}-\sum_{i j}\left(J_{i j}\left(S_{i}^{x} S_{j}^{x}+S_{i}^{y} S_{j}^{y}\right)+J_{i j}^{z} S_{i}^{z} S_{j}^{z}\right),
$$

where the couplings are defined by

$$
\left.\begin{array}{l}
J_{i j}=-\frac{p v\left(\zeta_{i}-\zeta_{j}\right)}{\sinh \left[p\left(v_{i}-v_{j}\right)\right]}, \\
J_{i j}^{z}=-p v\left(\zeta_{i}-\zeta_{j}\right) \operatorname{coth}\left[p\left(v_{i}-v_{j}\right)\right]
\end{array}\right\} i \neq j
$$

The eigenstates of $H_{S}^{x x z}$ in $\mathcal{H}_{S}$ and its eigenvalues are:

$$
\begin{aligned}
\left|\Phi_{N_{S}}^{x x z}\right\rangle & =\prod_{\alpha} \sum_{j} \frac{p S_{j}^{+}}{\sinh \left[p\left(\nu_{\alpha}-v_{j}\right)\right]}|0\rangle_{S}, \\
E_{N_{S}}^{x x z} & =p v \sum_{j \alpha} \zeta_{j} \operatorname{coth}\left[p\left(\nu_{\alpha}-v_{j}\right)\right]-\sum_{j} \zeta_{j}-\frac{1}{4} \sum_{i j} J_{i j}^{z}-\frac{1}{2} \sum_{i} J_{i i}
\end{aligned}
$$

where the $\nu_{\alpha}$ have to satisfy the RS equations

$$
\frac{2}{v}+\sum_{j} \frac{1}{v_{j}-\nu_{\alpha}}=2 \sum_{\beta}^{\prime} \frac{1}{\nu_{\beta}-\nu_{\alpha}} .
$$

In order to attack the Hamiltonian (11), i.e. having an XXX- instead of an XXZ-type coupling, we have to perform the isotropic limit for the Gaudin Hamiltonians and obtain $H_{S}^{x x x}=\sum_{i} 2 \zeta_{i} S_{i}^{z}-\sum_{i j} J_{i j} \vec{S}_{i} \cdot \vec{S}_{j}$, with the coupling 
defined as

$$
J_{i j}=-v \frac{\zeta_{i}-\zeta_{j}}{v_{i}-v_{j}} \quad, \quad i \neq j
$$

The eigenvalues and the eigenstates of $H_{S}^{x x x}$ are

$$
\begin{aligned}
\left|\Phi_{N_{S}}^{x x x}\right\rangle & =\prod_{\alpha} \sum_{j} \frac{c_{j \uparrow}^{\dagger} c_{j \downarrow}}{\nu_{\alpha}-v_{j}}|0\rangle_{S}, \\
E_{N_{S}}^{x x x} & =\sum_{j \alpha} \frac{v \zeta_{i}}{\nu_{\alpha}-v_{i}}-\sum_{j} \zeta_{j}-\frac{1}{4} \sum_{i j} J_{i j}-\frac{1}{2} \sum_{i} J_{i i},
\end{aligned}
$$

For uniform Zeeman splitting, $\zeta_{i} \equiv \mu_{B} B$ ( $\mu_{B}$ is Bohr's magneton, $B$ the magnetic field), the Hamiltonian can be expressed as

$$
H_{S}^{x x x}=2 \mu_{B} B S^{z}+\sum_{j}\left(2 \phi_{j} \Xi_{j}(\boldsymbol{S})-3 J_{j j} / 4\right) .
$$

We remark that the constants of motions are Gaudin Hamiltonians themselves (instead of the $\tau$ 's). The resulting couplings are given by Eq. (55) with $\zeta_{i}=\phi_{i}$. The eigenvalues of $H_{S}$ are then

$$
E_{N_{S}}=\mu_{B} B\left(2 N_{S}-\Omega_{S}\right)+\sum_{i \alpha} \frac{\phi_{i}}{\nu_{\alpha}-v_{i}}-\frac{1}{4} \sum_{i j} J_{i j}-\frac{1}{2} \sum_{i} J_{i i},
$$

where $\nu_{\alpha}$ have to be solutions of

$$
\sum_{j} \frac{1}{v_{j}-\nu_{\alpha}}=2 \sum_{\beta}^{\prime} \frac{1}{\nu_{\beta}-\nu_{\alpha}}
$$

The corresponding eigenstates are given by Eq.(56). The main difference is that the $\nu_{\alpha}$ fulfill the Gaudin Eqs.(60) instead of the RS Eqs.(54).

\section{Discussion of the coupling constants}

In the following we focus on possible choices of the coupling constants in $H_{K}$ that seem appropriate for the physics of metallic grains[23]. It is physically reasonable to demand that the pairing attraction decays with the energy difference of the interacting levels. This can be achieved by the choice $p=1$ in 


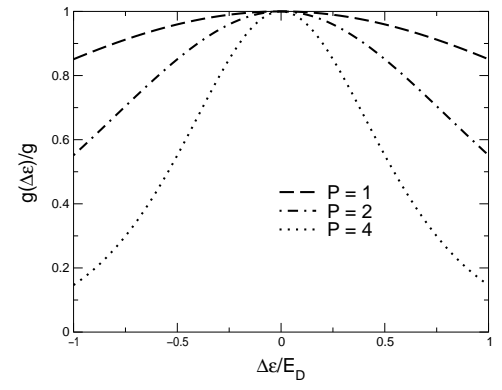

(a)

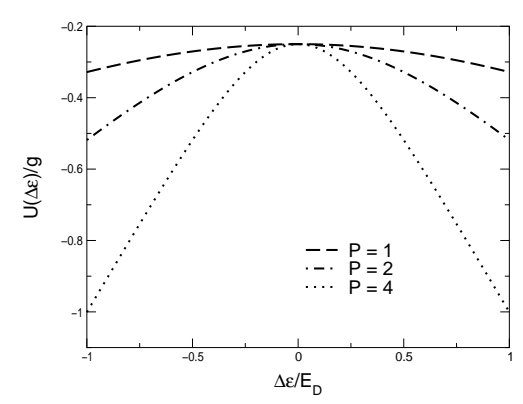

(b)

Fig. 1. (a) The pairing and (b) the Coulomb interaction as function of the energy difference $\Delta \varepsilon$ of the single-particle levels. In Figure (b) the $y$ axis is offset by $A / 4 g$ (see the definition of $U$ in Eqs. (20))

Eq.(20) — which corresponds to have hyperbolic functions in the couplings and $u_{i}$ being a monotonic function of the single particle energies. In order to make the couplings $g_{i j}$ and $U_{i j}$ depend on the energy difference $\varepsilon_{i}-\varepsilon_{j}$ alone, we choose $u_{j}=-\varepsilon_{j} / \delta \epsilon ; \delta \epsilon$ has the dimension of an energy ( $u_{i}$ are dimensionless), and it turns out to be a measure of the range of the pairing interaction. We introduce the dimensionless parameter $P$ such that $\delta \epsilon=E_{D} / P, 2 E_{D}$ being the width $\Delta \varepsilon_{\max }$ of the single particle spectrum. We choose $u=P g / E_{D}$, in order that the limit $P \longrightarrow 0$ corresponds to $g_{i j} \equiv g$. The resulting pairing is plotted in Fig.1(a). The Coulomb repulsion slowly decreases with the energy difference, as shown in Fig.1(b). For large energy difference and $P \geq 1$ the decay is linear in the single particle energy difference.

We now discuss the interpretation of Hamiltonian (8) as a model for $\mathcal{N}$ weakly coupled grains. We introduce the grain index $a=1, \ldots, \mathcal{N}$ (the index increasing with the spatial distance of the grains) and the sets $I_{a}$ such that for $i \in I_{a}, \varepsilon_{i}$ is an energy level of grain $a ; \bigcup_{a=1}^{\mathcal{N}} I_{a}=I$. The charge part e.g. of Hamiltonian (8) can then be rewritten as

$$
\begin{aligned}
H_{\mathcal{N}}= & \sum_{a=1}^{\mathcal{N}} \sum_{i_{a}} \xi_{i_{a}}^{(a)} c_{a, i_{a} \sigma}^{\dagger} c_{a, i_{a} \sigma}-\sum_{a, b=1}^{\mathcal{N}} \sum_{i_{a}, j_{b}} g_{i_{a} j_{b}}^{(a, b)} c_{a, i_{a} \uparrow}^{\dagger} c_{a, i_{a} \downarrow}^{\dagger} c_{b, j_{b} \downarrow} c_{b, j_{b} \uparrow} \\
& +\sum_{a, b=1}^{\mathcal{N}} \sum_{i_{a}, j_{b}} U_{i_{a} j_{b}}^{(a, b)} n_{a, i_{a} \sigma} n_{b, j_{b} \sigma^{\prime}}
\end{aligned}
$$

This Hamiltonian describes a system of many grains, where $i_{a}=1, \ldots \Omega_{a}$ labels the elements of $I_{a}$ and $c_{a, i_{a} \sigma}$ annihilates an electron with spin $\sigma$ in the $i_{a}$-th level of $a$-th grain, $\xi_{i_{a}}^{(a)}$. For $a \neq b, g^{(a, b)}$ describes the tunneling of Cooper pairs; $g_{i_{a} j_{b}}^{(a, b)}=g_{i j}$ if $i$ is the $i_{a}$-th element of $I_{a}$, and $j$ the $j_{b}$-th element of $I_{b}$; $U^{(a, b)}$ is the Coulomb repulsion between the grains $a$ and $b$. It is written in terms of $U_{i j}$ analogously to $g^{(a, b)}$. Couplings $g^{(a, a)}$ and $U^{(a, a)}$ describe intra- 


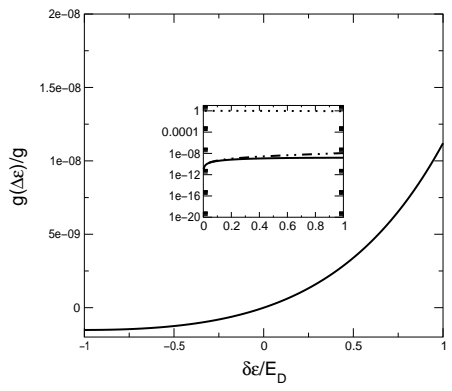

(a)

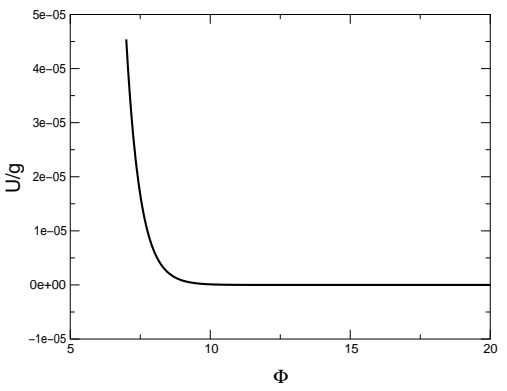

(b)

Fig. 2. (a) The inter-grain pairing coupling $g^{(1,2)}$, i.e. the pair-tunneling amplitude, between two grains with $P=1$ and $\Phi=\Phi_{2}-\Phi_{1}=20$. The inset shows that the pair tunneling amplitude $g^{(1,2)}$ is much smaller than $g^{(1,1)} \equiv g^{(2,2)}$. (b) The intra-grain Coulomb repulsion is nearly constant for $\Phi \gg P=1$ for fixed pairs of interacting levels; this constant increases linearly with $\Delta \varepsilon$ (the $y$ axis is offset by $A / 4 g$ ).

grain pairing and Coulomb interactions, respectively. Fixing the parameters as $u_{j}=\Phi_{a}-P \varepsilon_{j} / E_{D}$ for $j \in I_{a}$ and imposing $\Phi_{a+1}-\Phi_{a} \gg P$, the tunneling amplitude of pairs is exponentially suppressed compared to the intra-grain pairing coupling, as seen in Fig. 2(a).

\section{Conclusions}

In this paper, we have constructed a new class of integrable models as a functional of mutually commuting operators, which are a generalization of the BCS integrals of motion found in Ref. [12]. The latter are tightly connected with isotropic Gaudin Hamiltonians and the generalization presented in this paper consists in replacing isotropic with anisotropic Gaudin Hamiltonians. The exact solution of the Hamiltonian constructed in this way has been obtained via the diagonalization of these integrals of motion. This procedure is applied to two orthogonal realizations of $s u(2)$ (see section 2), leading to the model Hamiltonians $H_{K}$ and $H_{S}-$ Eqs. (18) and (19). The resulting models describe systems with nonuniform interactions, whose relevance was discussed in Ref. [24] according to recent experiments on metallic grains [1]. The nonuniform couplings we obtain are tunable by a set of parameters, which can be chosen such that physical demands on the couplings (see Ref. [6,7] and section 5) are fulfilled. This is demonstrated in Figs. 1 and 2 where the couplings are plotted for an exemplary choice of the parameters in the hyperbolic regime, $p=1$.

We also discuss possible applications of this class of models to systems of few coupled grains and present a choice of parameters such that the intra-grain couplings significantly dominate the inter-grain couplings. 
It is noteworthy that the class of Hamiltonians (18) and (19) can also describe a spin chain with a long-range, nonuniform XXZ interaction. Then, $\eta_{j}$ and $\zeta_{j}$ play the role of a nonuniform magnetic field and the indices $(i$ and $j)$ are to be interpreted as labeling lattice sites rather than energy levels. In this case the use of trigonometric functions ( $p=\mathrm{i}$ in Eqs. (20)) can account for boundary conditions.

We want to emphasize that also in nuclear physics [29,30], QCD [31], and astrophysics[32], the BCS Hamiltonian (with uniform couplings) has been thoroughly investigated. The three and four-body interactions, Eq.(38) and (39), might come in handy in these contexts.

Further generalizations of the present work seem interesting. Studies on the completely anisotropic XYZ Gaudin model can lead to Hamiltonians where the total number of particles (or the total $z$ component of the spin) is not conserved. These models are connected with the $2 D$ eight-vertex model $[25,26]$, and eventually there is a connection with its off-shell Bethe Ansatz alike that between the six-vertex model and the uniform BCS model [14,27].

In order to describe interacting bosons, su $(1,1)$ Gaudin models instead of $s u(2)$ ones must be considered; for the isotropic $s u(1,1)$ Gaudin model see Ref. [28]. Consequently, models based on anisotropic su $(1,1)$ Gaudin models should be studied as well.

Finally correlation functions for the present class of models can be studied along the lines described in Ref.[33].

\section{A Gaudin models}

Here we summarize the main results of Ref.[19], which are relevant for this work. Gaudin studied the following class of Hamiltonians:

$$
\tilde{\Xi}_{j}(\boldsymbol{\sigma})=\sum_{k}^{\prime} w_{j k}^{a} \sigma_{j}^{a} \sigma_{k}^{a}
$$

where $\sigma^{\alpha}$ are the Pauli matrices, and the sum over $a=x, y, z$ is implied. Mutual commutation of $\tilde{\Xi}_{j}$ requires

$$
w_{i j}^{a} w_{j k}^{c}+w_{j i}^{b} w_{i k}^{c}=w_{i k}^{a} w_{j k}^{b} .
$$

Identifying $(x, y, z)$ with $(1,2,3)$, let us define

$$
w_{i j}^{a}=: \frac{\Theta^{a+1}\left(u_{i j}\right)}{\Theta^{a+1}(0) \Theta^{1}\left(u_{i j}\right)},
$$


with $u_{i j}=-u_{j i} ; \Theta^{1}(u)$ is an odd function, and $\Theta^{a+1}(u)$ are three even functions. For $u_{i j}$ such that $u_{i j}+u_{j k}+u_{k i}=0$ (e.g. $u_{i j}=u_{i}-u_{j}$ ), it is found that Eqs.(A.2) are satisfied if $\Theta^{a}$ are the elliptic Jacobi functions. It is possible to find eigenstates and eigenvalues of $\tilde{\Xi}_{i}$ through Bethe Ansatz[19,20].

Imposing conservation of the $z$-projection of the total spin, $\left[\sum_{i} \sigma_{i}^{z}, \tilde{\Xi}_{j}\right]=0$, we get the conditions $w_{j k}^{1}=w_{j k}^{2}$, i.e. the anisotropy is of XXZ-type. In this case, the (rescaled) elliptic functions reduce to the trigonometric ones

$$
\begin{aligned}
w_{i j} & =w_{i j}^{1}=w_{i j}^{2}=p w / \sinh \left[p\left(u_{i}-u_{j}\right)\right] \\
v_{i j} & =w_{i j}^{3}=p w \operatorname{coth}\left[p\left(u_{i}-u_{j}\right)\right] .
\end{aligned}
$$

Here, we included a real scale factor $w$ together with the parameter $p$ which can either be real or imaginary. Defining

$$
\tilde{\sigma}^{ \pm}(u)=\sum_{i} \frac{p w}{\sinh \left[p\left(u_{i}-u\right)\right]} \sigma_{i}^{ \pm}, \quad \tilde{\sigma}^{z}(u)=\sum_{i} p w \operatorname{coth}\left[p\left(u_{i}-u\right)\right] \sigma_{i}^{z}
$$

the common eigenstates $\left|\Psi_{N_{\uparrow}}\right\rangle$ of $\tilde{\Xi}_{i}$ for $N_{\uparrow}$ up spins read

$$
\left|\Psi_{N_{\uparrow}}\right\rangle=\prod_{\alpha} \tilde{\sigma}^{+}\left(\omega_{\alpha}\right)|0\rangle
$$

where $|0\rangle$ is the lowest weight state. The corresponding eigenvalues are

$$
\tilde{\xi}_{i}=\sum_{j}^{\prime} p w \operatorname{coth}\left[p\left(u_{i}-u_{j}\right)\right]-2 \sum_{\alpha} p w \operatorname{coth}\left[p\left(u_{i}-\omega_{\alpha}\right)\right]
$$

where $\omega_{\alpha}$ must satisfy

$$
\sum_{j} p \operatorname{coth} p\left[\left(u_{j}-\omega_{\alpha}\right)\right]=2 \sum_{\beta}^{\prime} p \operatorname{coth}\left[p\left(\omega_{\beta}-\omega_{\alpha}\right)\right]
$$

$\alpha, \beta$ run from 1 to $N_{\uparrow}$. The isotropic case is obtained imposing $\left[\boldsymbol{S}, \Xi_{i}\right]=0$, and corresponds to the limit $p \rightarrow 0$. It leads to the isotropic Gaudin Hamiltonians

$$
\Xi_{j}(\boldsymbol{\sigma})=\sum_{k}^{\prime} \frac{\vec{\sigma}_{j} \cdot \vec{\sigma}_{k}}{u_{j}-u_{k}}
$$

Using here the operators

$$
\sigma^{ \pm}(u)=\sum_{i} \frac{\sigma_{i}^{ \pm}}{\left(u_{i}-u\right)}, \quad \sigma^{z}(u)=\sum_{i} \frac{\sigma_{i}^{ \pm}}{\left(u_{i}-u\right)},
$$


the common eigenstates of $\Xi_{i}$ for $N_{\uparrow}$ up spins can be written as:

$$
\left|\Psi_{N_{\uparrow}}\right\rangle=\prod_{\alpha} \sigma^{+}\left(\omega_{\alpha}\right)|0\rangle
$$

and the corresponding eigenvalues are:

$$
\xi_{i}=\sum_{j}^{\prime} \frac{1}{u_{i}-u_{j}}-2 \sum_{\alpha} \frac{1}{u_{i}-\omega_{\alpha}},
$$

where $\omega_{\alpha}$ have to satisfy

$$
\sum_{j} \frac{1}{u_{j}-\omega_{\alpha}}=2 \sum_{\beta}^{\prime} \frac{1}{\omega_{\beta}-\omega_{\alpha}}
$$

We note, that the specialized Gaudin equations (A.13) and their more general version (A.8) are the $g \rightarrow \infty$ limit of the RS equations (17) and their generalization (43), respectively.

\section{References}

[1] C.T. Black, D.C. Ralph, and M. Tinkham, Phys. Rev. Lett. 74, 3291 (1995); 76, 688 (1996); 78, 4087 (1997).

[2] J. von Delft and D.C. Ralph, Phys. Rep. 345, 61 (2001).

[3] D.G. Salinas, S. Guéron, D.C. Ralph, C.T. Black, and M. Tinkham, Phys. Rev. B 60, 6137 (1999); D. Davidovic and M. Tinkham, Phys. Rev. Lett. 83, 1644 (1999).

[4] S. Guéron, M.M. Deshmukh, E.B. Myers, and D.C. Ralph, Phys. Rev. Lett. 72, 3861998.

[5] A. Di Lorenzo, R. Fazio, F. Hekking, G. Falci, and A. Mastellone, Phys. Rev. Lett. 84, 550 (2000); M. Schechter, Y. Imry, Y. Levinson, and J. von Delft Phys. Rev. B 63, 214518 (2001).

[6] L. I. Glazman in Ref. [10].

[7] I.L. Kurland, I.L. Aleiner, and B.L. Altshuler, Phys. Rev. B 62, 14886 (2000).

[8] P. Jacquod, A. Douglas Stone, cond-mat/0102100.

[9] Y. Alhassid, Rev. Mod. Phys. 72, 895 (2000).

[10] Proceedings of the International Conference on Electron Transport in Mesoscopic Systems, ETMS'99 J. Low Temp. Phys. 118 (2000). 
[11] R.W. Richardson and N. Sherman, Nucl. Phys. 52, 221 (1964); 52, 253 (1964).

[12] M.C. Cambiaggio, A.M.F. Rivas, and M. Saraceno, Nucl. Phys. A 624, 157 (1997).

[13] L. Amico, A. De Lorenzo, and A. Osterloh, Phys. Rev. Lett. 86, 5759 (2001).

[14] L. Amico, G. Falci, and R. Fazio, J. Phys. A 34, 6425 (2001).

[15] G. Sierra, J. Dukelsky, G.G. Dussel, J. von Delft, and F. Braun, Phys. Rev. B 18, R11890 (2000).

[16] O. Agam, N.S. Wingreen, B.L. Altshuler, D.C. Ralph, and M. Tinkham, Phys. Rev. Lett. 78, 1956 (1997); B.L. Altshuler, Y. Gefeln, A. Kamenev, and L.S. Levitov, Phys. Rev. Lett. 78, 2803 (1997); Ya.M. Blanter, Phys. Rev. B 54, 12807 (1996).

[17] The only extension we found in this way, was a simply "rotated" model, where each eigenstate was rotated by a phase. In this case, the (complex) pairing coupling had the simple form $g_{j k}=g \operatorname{expi}\left(\varphi_{j}-\varphi_{k}\right)$. The RS equations are consequently unchanged.

[18] F. Braun and J. von Delft, Phys. Rev. Lett. 81, 4712 (1998).

[19] M. Gaudin, J. Phys. 37, 1087 (1976).

[20] E.K. Sklyanin, J. Sov. Math. 47, 2473 (1989).

[21] It was not included in $u,\left\{u_{j}\right\}$ in order to make clear the relation with the isotropic case, obtained in the limit $p \rightarrow 0$.

[22] In this case, the contributions from Hamiltonians $H_{3}$ and $H_{4}$ do not vanish. Nevertheless, these terms might be useful to describe a system of neutral particles with three and four-body interactions.

[23] The possible range of couplings is very large: there are as many free parameters as single-particle levels. In $H_{S}$ for constant Zeeman splitting, the range is even wider, because the $\phi_{j}$ are an additional set of independent parameters. We do not show an exemplary plot of the spin couplings.

[24] U. Sivan, F.P. Milliken, K. Milkove, S. Rishton, Y. Lee, J.M. Hong, V. Boegli, D. Kern, and M. deFranza, Europhys. Lett. 25, 605 (1994), O. Agam, in Supersymmetry and Trace Formulae: Chaos and Disorder, I. V. Lerner, J. P. Keating and D. E. Khmelnitskyi editors, Plenum Press 1999..

[25] M. Gaudin, La fonction d'onde de Bethe, (Masson, Paris 1982).

[26] E.K. Sklyanin and T. Takebe, Phys. Lett. A 219, 217 (1996).

[27] H.M. Babujian, J. Phys. A 26, 6981 (1993); H.M. Babujian and R. Flume, Mod. Phys. Lett. 9, 2029 (1994).

[28] J. Dukelsky and P. Schuck Phys.Rev.Lett. 86, 4207 (2001).

[29] F. Iachello, Nucl. Phys. A 570, 145c (1994). 
[30] R.W. Richardson, private communication.

[31] D. H. Rischke and R. D. Pisarski, proceedings of the "Fifth Workshop on QCD", Villefranche, nucl-th/0004016.

[32] H. Heiselberg and M. Hjorth-Jensen, Phys. Rep. 328, 237 (2000).

[33] L. Amico and A. Osterloh, submitted to Phys. Rev. Lett., cond-mat/0105141. 